\section{LA PREOCUPANTE FALTA DE FORMACIÓN EN DESASTRES DENTRO DE LAS ESCUELAS DE MEDICINA LATINOAMERICANAS}

De acuerdo al Centro de Investigación sobre la Epidemiología de los Desastres, entre 1974 y 2003 ocurrieron 6367 desastres en el mundo, sin contar las epidemias. Estos desastres han dejado un saldo de más de 2 millones de muertos, 5100 millones de afectados, 182 millones de personas sin hogar y daños en infraestructura valuados en US\$ 1,38 billones (1).

Solo en el presente año, dos devastadores terremotos asolaron a nuestro continente, uno el 12 de enero en Haití, que dejó un saldo aproximado de 230000 muertes, 300000 lesionados y 1000000 de personas sin hogar, y otro el 28 de febrero en Chile, donde se notificaron 342 víctimas fatales, 97 desaparecidos, 800000 damnificados y pérdidas materiales por unos US\$ 29662 millones $(2,3)$. En 2005, el huracán Katrina dejó a su paso una ciudad prácticamente destruida por las inundaciones, más de 1800 personas muertas y cerca de 146000 sin hogar, y daños materiales por US\$ 75000 millones, en lo que se ha considerado la mayor catástrofe natural de Estados Unidos (4).

Se prevé que el número de desastres naturales -incluidos aludes, erupciones volcánicas, huracanes, inundaciones, maremotos y terremotos-y su impacto proporcional en la gente aumentarán debido a los cambios climáticos, al desplazamiento de poblaciones a zonas de mayor exposición y al aumento de la densidad poblacional. Su ocurrencia genera problemas de salud a corto, mediano y largo plazo. Dentro de la primera fase se encuentran el rescate, la atención de heridos con lesiones de diversa gravedad y el manejo de cadáveres. En la segunda sobreviene un mayor riesgo de enfermedades transmisibles, que crece en función del hacinamiento y el deterioro de la situación sanitaria, los daños sufridos por los establecimientos de salud y el desabastecimiento de agua potable, alimentos y otros bienes básicos (5).

En el largo plazo adquieren particular relevancia las consecuencias psicológicas del desastre. Dentro de las más frecuentes se encuentran el trastorno por estrés post traumático (TEPT), la depresión mayor, el aumento de consumo de drogas y alcohol, otros trastornos de ansiedad y los síntomas de somatización (6). Durante el primer año post terremoto en Chile, 30 a $40 \%$ de los damnificados, 10 a $20 \%$ de los intervinientes y 5 a $10 \%$ de la población general podrían presentar TEPT (7). Un tercio de los pacientes con TEPT continuará sintomático una década después, con altísimos costos por ausentismo laboral y suicidio (8).

De los tres componentes principales - preparación, mitigación y respuesta- que conforman la ges- tión de la salud en casos de desastre, la preparación reviste una importancia fundamental $(5,6)$. En este sentido, el personal sanitario debería contar con las capacidades técnicas necesarias en todos los tópicos relativos a los desastres, desde la prevención a la respuesta y luego a las consecuencias sobre la población en sus diversas fases. La gestión en desastres tendría entonces que formar parte de los programas de estudios de la Región a nivel de pre y post grado, comenzando por las escuelas de medicina, de enfermería y de salud ambiental (5).

Con objeto de informarnos sobre este tema, examinamos los planes de estudios de pregrado para la carrera de médico-cirujano de las 37 universidades hispanoparlantes que contaban con escuelas de medicina y conformaban el grupo de las 100 mejores universidades latinoamericanas (9). Hallamos que solo una $(2,7 \%)$ incluía algún grado de formación en situaciones de desastre, una realidad preocupante que debe ser encarada tanto por las autoridades sanitarias como por las casas de altos estudios y otros centros de formación en salud.

Es preciso y prioritario que las escuelas de medicina incorporen en sus planes de estudios un programa integral sobre desastres, donde se revisen aspectos de prevención, organización - nacional y local- y respuesta frente a estas emergencias, así como el manejo de sus consecuencias sanitarias a corto, mediano y largo plazo. Los desastres constituyen un problema de salud pública de alta relevancia. Todos estamos expuestos a ellos $\mathrm{y}$, aunque no sabemos cuándo, sí tenemos certeza de que ocurrirán, por lo que resulta fundamental que nuestros profesionales de la salud se encuentren adecuadamente preparados para enfrentarlos.

Luigi Accatino

Escuela de Medicina

Pontificia Universidad Católica de Chile Santiago, Chile

Rodrigo A. Figueroa Unidad de Trauma, Estrés y Desastres Departamento de Psiquiatría Escuela de Medicina

Pontificia Universidad Católica de Chile

Joaquín Montero Departamento de Medicina Familiar Escuela de Medicina

Pontificia Universidad Católica de Chile

Matías González

Departamento de Psiquiatría Escuela de Medicina

Pontificia Universidad Católica de Chile Correo electrónico: magonza@med.puc.cl 


\section{REFERENCIAS}

1. Guha-Sapir D, Hargitt D, Hoyois P. Thirty Years of Natural Disasters 1974-2003: The Numbers. Louvian, Belgium: Presses universitaires de Louvain; 2004.

2. Terremoto de Haití 2010. Hallado en: http://es.wikipedia. org/wiki/Terremoto_de_Hait\%C3\%AD_de_2010. Acceso el 03 de septiembre de 2010.

3. Diario "El Mercurio". Terremoto en Chile [cobertura especial]. Hallado en: http://www.emol.com/especiales/2010/ coberturas-especiales/terremoto-en-chile/cifras.html. Acceso el 03 de septiembre de 2010.

4. Huracán Katrina. Hallado en: http://es.wikipedia.org/wiki/ Katrina. Acceso el 03 de septiembre de 2010.
5. Organización Panamericana de la Salud (OPS). Los desastres naturales y la protección de la salud. Washington D.C.: OPS; 2000.

6. Figueroa RA, Marín H, González M. Psychological support for disaster victims: An evidence-based care model. Rev Med Chil. 2010;138(2):143-51.

7. Galea S, Nandi A, Vlahov D. The epidemiology of post-traumatic stress disorder after disasters. Epidemiol Rev. 2005;27:78-91.

8. Green BL, Lindy JD, Grace MC, Leonard AC. Chronic posttraumatic stress disorder and diagnostic comorbidity in a disaster sample. J Nerv Ment Dis. 1992;180(12):760-6.

9. Consejo Superior de Investigaciones Científicas de España. Ranking Web de Universidades del Mundo. Top Latino Americano. Hallado en: http://www.webometrics.info/top100_continent es.asp?cont=latin_america. Acceso el 03 de septiembre de 2010. 